\title{
INVESTIGATION OF THE RESISTANCE A SAILBOAT IS SUBJECTED TO IN THE CASE OF DRAFT CHANGES CAUSED BY MODIFYING THE POW- ERTRAIN
}

\author{
EMESE LÉVAI*1 AND PÉter FICZERE ${ }^{1}$ \\ ${ }^{1}$ Department of Railway Vehicles and Vehicle System Analysis, Budapest University of Technology and \\ Economics, Múegyetem rkp. 3, Budapest, 1111, HUNGARY
}

\begin{abstract}
In our research, calculations were performed using the example of a specific ship when a modification was made to the powertrain of a vehicle. These results were used to observe whether the ship is expected to decelerate or the change in the draft is likely to cause stability problems. In recent years, the rules concerning the use of inland waterways by recreational crafts have been tightened for environmental reasons. In many cases, these restrictions affect the drive chain, making the workflow of the conversion involved frequent. In this case, it is extremely important for shipowners to know how the draft will change and at what speed the ship will operate after the conversion. This study on a numerical flow simulation provides an excellent opportunity to find out.
\end{abstract}

Keywords: sailing, computational fluid dynamics, simulation, modifying the powertrain

\section{Introduction}

With the boom in lake navigation, the technical equipment of small watercraft has also expanded to include important details. Since the number of sailing vessels, which were originally only wind-powered, has increased in ports, in order to prevent subsequent disruptions and facilitate emergency maneuvering, these vessels carry an engine as a secondary source of propulsion. In addition, small boats that are purely motor-powered have also entered the market for private individuals. Generally speaking, this size of engine has had a detrimental effect on life in shallow lakes, such as Lake Balaton, in several ways and emissions have noticeably increased. As a result, a regulation [1] came into force whereby recreational (i.e., not emergency, port maneuvering, etc.) trips can only be powered by electric motors on Lake Balaton. For this reason, shipowners have had to convert their propulsion systems from internal combustion engines to electric versions.

Although the effect of this on the draft and speed varies from boat to boat, this data is important for shipowners. Our article presents a method for calculating such data for a specific ship.

*Correspondence: levai.emese@edu.bme.hu

\section{Methods}

\subsection{Weight and dive calculation}

The examined vessel is a $50 \mathrm{~m}^{2}$ cruising, capitalweighted sailboat designed for tours that is $13.2 \mathrm{~m}$ long and weighs $6.8 \mathrm{t}$ (Figs. 1 and 2). The differences between the results of the weight calculations on the blueprints and the actual ship, as well as the knowledge of the mass of the elements of the electric and diesel powertrain, were sufficient to determine the change in the draft [2]. The draft varies from $1.68 \mathrm{~m}$ to $1.7 \mathrm{~m}$ (Fig. 3). The calculation was performed using the MAXSURF Hydromax program.

\subsection{Fluid dynamics simulation}

The purpose of the calculation in this case is twofold: to determine the drag (resistance) force acting on the hull while in motion (' $x$ ' component) and the buoyancy (' $z$ ' component). The practical goal of the tests is to calculate the resistance force acting on the boat during two different dives using the same engine power, as this has a major impact on the speed of travel (because if the boat moves in the ' $x$ ' direction, the resistance force will always be in the ' $x$ ' direction). The tests were performed using the FloEFD extension of the Siemens Solid Edge program. The initial values were the dive $(1.68 \mathrm{~m}$ and 1.7 $\mathrm{m}$ according to the weight calculation) (Fig. 3), flow characteristics - test, free surface, and the boundary between 


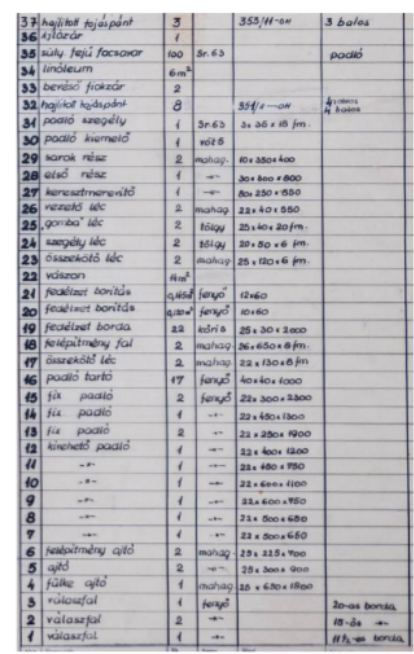

Figure 1: A piece of the original item and weight chart (1950)

different media, as well as their required properties (e.g., density, method, etc.). As an external force, the magnitude and direction of gravity had to be taken into account. The flow rate of the tested medium was also adjusted to the medium in the appropriate direction, that is, opposite to the direction of travel. The mesh was compressed locally by 3 -stage compression around the surfaces delimiting the body (Figs. 4 and 5).

This is partly due to the fact that unnecessarily accurate calculations of values at points unrelated to the hull would lengthen the duration of the test and partly the result of the calculation being as accurate as possible at critical locations (where plating and water intersect). The load cases were chosen according to the dives and the selected speed points at a Froude number of up to 0.45 can be assigned to the characteristic operating conditions of the ship (while displacing water) [3]. The values at the selected speed points were recorded within this range.

\section{Results}

After running the analysis, the series of measurements was tested separately for each load case at each of the six speed points. Convergence was observed under all circumstances. The results, which are shown in Figs. 6 and 7 , were plotted on graphs and presented in tables. It can be seen that in both the ' $x$ ' and ' $z$ ' direction, the higher the speed at which the vessel travels, the greater both the force acting on the hull and the greater the vertical distances measured between the points of the curve on the graphs are. As can be seen in Fig. 4, the resistance (vertical axis) resulting from lighter load cases (denoted by the orange line) was less than for heavier load cases when the electric drive chain was in use (denoted by the gray line). The expected results were also recorded in the evaluation of the buoyancy forces. Regarding the load case belonging to the original drive chain (denoted by the gray line), the buoyancy forces are lower than in terms of the load

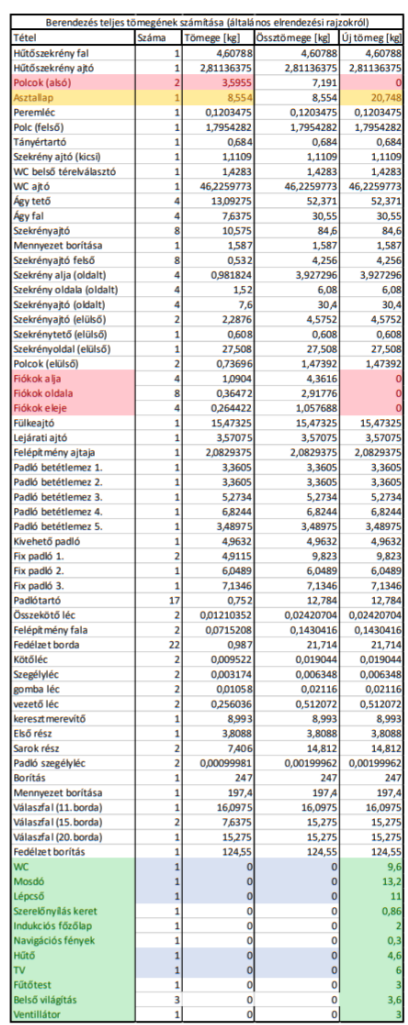

Figure 2: A portion of the new batch and weight chart (2020, field survey)

case belonging to the new drive chain (denoted by the blue line). The parabolic nature of the curves was also in line with the preliminary expectations as the resistance and velocity are square proportional. On the curve depicting the resistance, the wavy nature (at a Froude number of approximately 0.5 ) is due to the effect of the wave resistance on the total resistance.

This also means that even as a result of small changes in dive, the increase in resistance becomes more significant as the vessel accelerates. Therefore, even on a larger ship, it might be beneficial to select lighter components for the powertrain.

Not only does dive cropping change the size of the wetted surface and thus the resistance force, the shape of the wetted surface and the waterline section is also modified. In the present case, it geometrically cuts a wider shape out of the water surface as a result of the hull, so

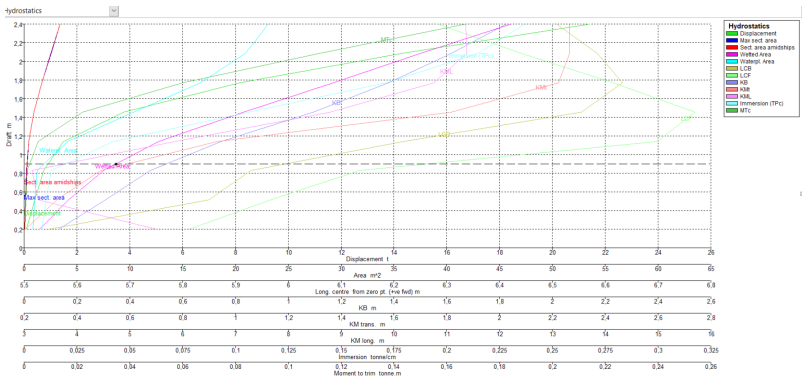

Figure 3: Characteristic curves calculated from vessel data 


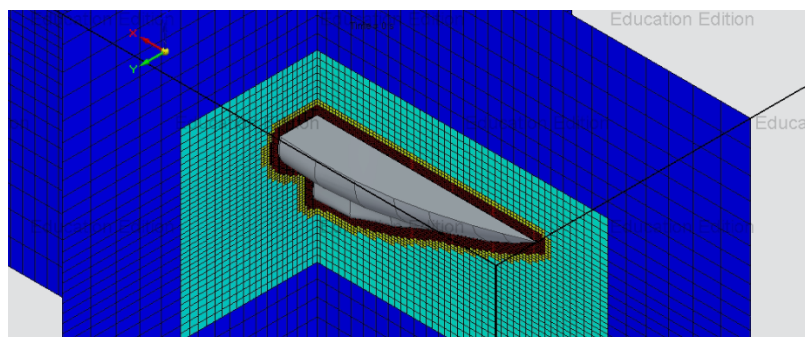

Figure 4: Locally compressed mesh around the stern of the hull

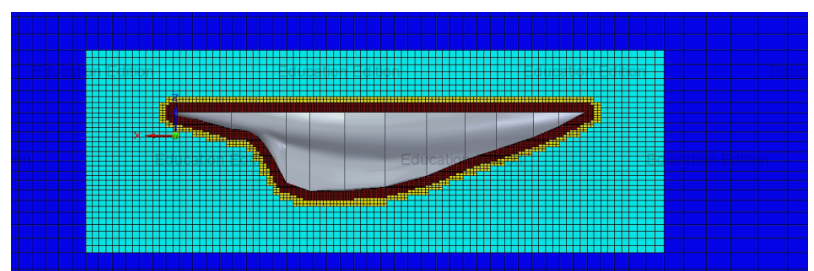

Figure 5: Locally compressed mesh around the stern of the hull (side view)

the current image must also be examined (Fig. 7). If the immersion shape changes leading to the flow rate generating early-breaking vortices around the hull or accelerating too quickly around the maximum width, a drastic reduction in speed results.

\section{Analysis}

By examining the results, several findings are made. At higher speeds, as was expected, the resistance force on the ship differs greatly between the two dives, increasing the draft from $1.68 \mathrm{~m}$ to $1.7 \mathrm{~m}$ by $4606 \mathrm{~N}$. Therefore, the effect of changing the draft in the order of a few centimeters is also significant. By plotting the velocity distribution around the vessel from the current image, it was found that behind the point where the width of the vessel is greatest (towards the stern) next to the side plate, the flow rate accelerates locally and then decelerates again back to the velocity observed around the front of the vessel. Around the ship, in addition to the surface that is in the shadow of the overflow (i.e., the accelerated flowlines next to the ship do not-or only partially-affect), the velocity of the medium decreases significantly. The velocity of the medium at and around the intersection of the waterline area and the axis of the steering bearing is close to $0 \mathrm{~m} / \mathrm{s}$. It can be seen that neither the magnitude $(13.7 \mathrm{~m} / \mathrm{s})$ nor the location of the maximum flow velocity (the narrowing arc behind the main rib) causes a large decrease in velocity (Fig. 8).

\section{Conclusion}

It can be said that by replacing the internal combustion engine and its associated drive chain, this ship will be subjected to an excessive resistance force which will reduce its forward speed. The test vessel is mass-produced

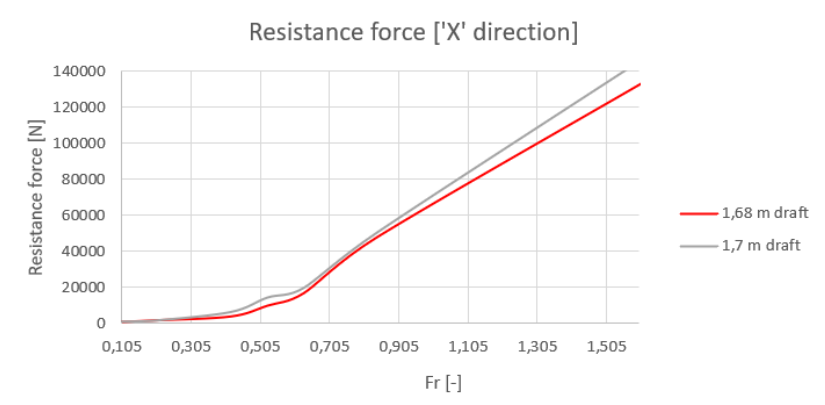

Figure 6: Comparison of the magnitude of the resistance a ship is subjected to under two load cases

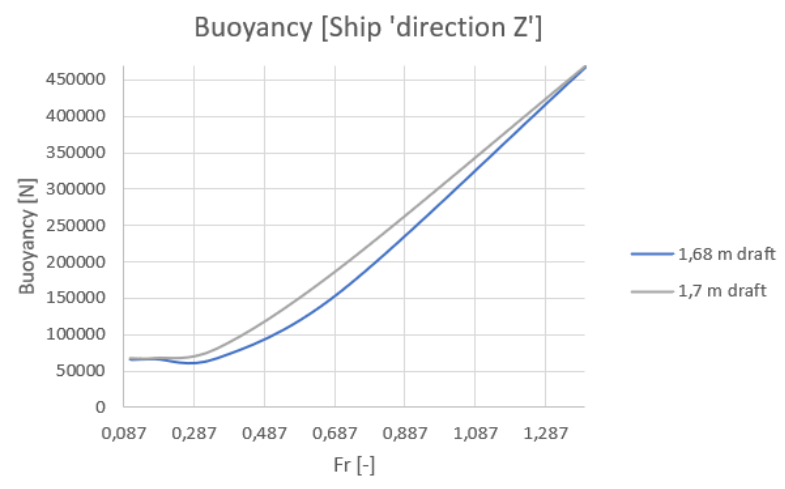

Figure 7: Comparison of the magnitude of the buoyancy acting on a ship under two load cases

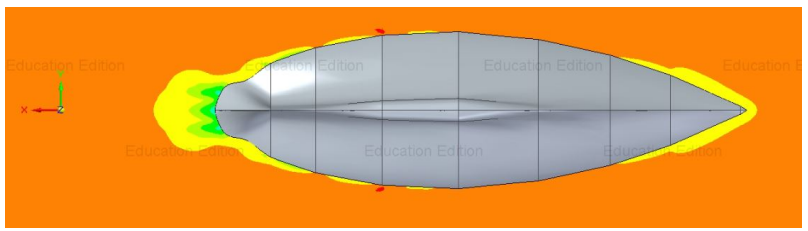

Figure 8: Flow velocity around the ship (higher: red, lower: orange)

and, in the case of vessels operating with possible minor modifications to its class, the calculation is expected to yield the same result with similar engines. For other types of vessels, by following the testing methodology, accurate answers to the questions raised in Section 2.2 are provided, which are vital, for example, before a sailing race (in which case, the engine is merely excess ballast). Since the calculations were performed on a horizontally floating sailboat, the subject of a further study could be the examination of a tilted vessel or of a hull protruding whilst accelerating using the same methodology. Questions may also be raised about an excessive number of additional batteries that may be inserted to increase the range of the electric motor and their possible placement in the light of swimmers as this presupposes additional dive options.

\section{REFERENCES}

[1] XLII/2000,Water Transport Act

[2] Lévai, E.: Elektromos segédhajtás beépítése egy acél építési anyagú, $50 \mathrm{~m}^{2}$-es tengeri cirkáló típusú, 
kedvtelési célú vitorlás kishajóba, 2021, 2-21, 3035

2012, 14-15 ISBN: 978-963-279-643-7

[3] Simongáti, Gy.; Hargitai, L.: Kishajók. Budapest, 